\title{
An application of item response theory to psychological test development
}

\author{
Cristian Zanon ${ }^{1 *}$, Claudio S. Hutz ${ }^{2}$, Hanwook (Henry) Yoo ${ }^{3}$ and Ronald K. Hambleton ${ }^{3}$
}

\begin{abstract}
Item response theory (IRT) has become a popular methodological framework for modeling response data from assessments in education and health; however, its use is not widespread among psychologists. This paper aims to provide a didactic application of IRT and to highlight some of these advantages for psychological test development. IRT was applied to two scales (a positive and a negative affect scale) of a self-report test. Respondents were 853 university students ( $57 \%$ women) between the ages of 17 and 35 and who answered the scales. IRT analyses revealed that the positive affect scale has items with moderate discrimination and are measuring respondents below the average score more effectively. The negative affect scale also presented items with moderate discrimination and are evaluating respondents across the trait continuum; however, with much less precision. Some features of IRT are used to show how such results can improve the measurement of the scales. The authors illustrate and emphasize how knowledge of the features of IRT may allow test makers to refine and increase the validity and reliability of other psychological measures.
\end{abstract}

Keywords: Item response theory, Graded response model, Psychological assessment, Affects

\section{Background}

Valid and reliable measures are essential to the field of psychology, as well as, to the study of abilities, aptitudes, and attitudes. In this sense, classical test theory (CTT) has been extensively serving the testing field for about 100 years. However, the implementation of item response theory (IRT) to psychological and educational assessment has caused major and positive changes to psychological test development (see, for example, Hambleton \& Jodoin, 2003). The use of IRT with test development has several advantages over CTT mainly because IRT produces person parameter invariance (test scores are not dependent on the particular choice of test items) when model fit is present, and test information functions provide the amount of information or "measurement precision" captured by the test on the scale measuring the construct of interest (Embretson, 1996; Hambleton et al. 2000) and other features too. These features and others along with CTT drawbacks, have led the authors of this paper to introduce Samejima`s (1969) graded response model

\footnotetext{
* Correspondence: crstn.zan@gmail.com

'Department of Psychology, Universidade São Francisco, Alexandre

Rodrigues Barbosa Str. 45, Itatiba 13251-900SP, Brazil

Full list of author information is available at the end of the article
}

(GRM). Estimation of latent ability using a response pattern of graded scores. Psychometrika (1969) graded response model (GRM), the topic of model fit, and other important characteristics of IRT analysis, to the development of psychological tests. In addition, another goal of this paper is to present a comprehensible application of the GRM, to interpret these results, and to highlight the importance of the findings provided by these analyses for psychological test development.

The topics regarding CTT, IRT, item and ability parameter invariance, IRT assumptions, the GRM and model fit will be presented and explained. The remainder of the paper will introduce an application of the GRM to our data, the methodology used, and how these results can be considered in order to refine the Affect Scale.

\section{Classical test theory}

As Gulliksen (1950) presented, and many readers know, within a CTT framework, item statistics are examinee sample-dependent for CTT models. This means that test item statistics are very dependent on the sample of examinees used in item calibration. But it would facilitate test development if the item statistics were not directly tied to the choice of examinee sample. In a similar way, 
examinee test scores too are dependent on the choice of test items. This means, for example, for examinees to be compared, they need to either take the same test items, or statistically equivalent test items. But there are times when better testing would result if the choice of test items was matched to the examinees' ability levels. Examinees scoring high on a construct might receive more challenging items, for example. But individualing the choice of test items, would limit the possibility of comparing examinees. Next, four limitations of CTT are presented (and for another perspective, see Embretson, 1996).

The first shortcoming of CTT is that the interpretation of respondent characteristics depend on the test used. Respondents will appear smarter, if an easier test is administered, but will look like less smart, if a more difficult test is answered. The second shortcoming of CTT is that test characteristics are sample-dependent. The same test administered in a group of high ability students and in another group of low ability students will produce items with different levels of difficulty, for example. In the first sample, items difficulty will appear lower than the difficulty for the second group. These shortcomings imply that test characteristics can only be done in the same context (sample). Once, test parameters depend on persons' latent trait and vice versa, item and test characteristics will change when other persons (samples with different levels of latent trait) answer the test.

The third shortcoming of CTT is that the theory assumes that errors of measurement are equal for all persons. This is problematic because persons with different levels of ability will show different levels of error (guessing) in a test that evaluates intelligence or any other construct, for example. The fourth shortcoming of CTT is that it does not allow accurate predictions about possible results for a respondent or for a sample on an item, using only their ability scores. This information would be important for a test designer interested in developing a test for a population with specific characteristics. All these points can be addressed effectively with IRT, if of course an IRT model can be found that fits the test data. ${ }^{1}$

\section{Item response theory development and properties}

IRT was initially developed in the 1950s and 1960s by Frederic Lord and other psychometricians (Lord, 1952; Lord \& Novick, 1968) who had the goal of developing a method able to evaluate respondents without depending on the same items included in the test (Hambleton \& Jodoin, 2003). Therefore, IRT evolved from classical measurement theory with the purpose of overcoming many of its limitations (Hambleton, 1994). IRT is a statistical theory comprised of a variety of mathematical models that have the following characteristics: a) to predict person scores based on his/her abilities or latent traits and b) to establish a relationship between person's item performance and the set of traits underling item performance through a function called the "item characteristic curve" (Hambleton et al. 1991). These characteristics are possible because IRT models provide item and ability parameter invariance for test items and persons, when the IRT model of interest actually fits the available test data. In other words, the same items used in different samples will keep their statistical properties (for instance, difficulty and discrimination), and persons' scores that represent ability or latent traits on a specific construct will not depend on the particular test items they were administered.

\section{The role of item and person parameter invariance in IRT}

Parameter invariance is not a mysterious property that IRT confers to items and tests. It refers to population quantities, rather than sample quantities, which are assumed to be identical across groups or measurement conditions (Rupp \& Zumbo, 2004). ${ }^{2}$ Parameter invariance is not exclusively found on IRT models. For instance, multiple regression analysis may also show such properties, once the residuals (distance between observed and expected scores) are considered small enough to assume that the equation which builds the model fits equally well data from different populations (Hambleton, 2005). If parameter variance is obtained in different populations, inferences and generalizability of the results will be threatened and probably be misleading (Rupp \& Zumbo, 2004).

Conceptually, it is not assumed that the same scores will be found if one person responds twice to the same test (Lord and F. M. A theory of test scores. Psychometrika 1952) because of learning, motivation level, fatigue effects, and other measurement errors. However, it is assumed that a respondent with similar latent traits will obtain similar scores (apart from measurement errors) on the same or on a completely different test built to evaluate the same construct. In addition, persons with different latent traits will preserve the same difference among their scores, if they answered a parallel form of the test.

\section{IRT assumptions}

There are two basic assumptions that pertain to all unidimensional ${ }^{3}$ IRT applications: unidimensionality and the shape of the item characteristic curves (ICC) (Hambleton et al., 1991). According to these authors, it is assumed that only one latent construct is measured by a set of items in a test. Therefore, the presence of a dominant factor explaining most of the instrument variance scores is expected. Some common techniques used for checking unidimensionality are: eigenvalue plots $(20 \%$ or more variability on the first factor: Hattie, 1985), parallel analysis (Horn, 1965) or confirmatory factor analysis (testing 
the hypothesis of a single factor). Other new recommended techniques, although not very often used, to evaluate unidimensionality are the Tetrad analysis and the Hull method (Yu et al. 2007; Lorenzo-Seva et al. 2011). We suggest readers interested in test unidimensionality see Hattie (1985) for a deep review about the topic.

The second assumption is that ICC, specified by the model, reflect the relationships between the latent traits and the item responses. When the test items are binary scored (e.g., true-false, or yes-no), ICCs tend to be Sshaped curves and match the probabilities of examinees successfully answering a test item as a function of their ability. Test items that are easy are shifted to the left on the scale measuring the trait, and items that are hard are shifted to the right end of the measuring scale. Discriminating items have higher slopes than lower discriminating items. With appropriate model fit, the ICC match up closely to the actual test data.

\section{PLM, 2PLM and 3PLM: models for binary data}

Three common IRT models for binary data are the one, two and three parameter logistic models. They differentiate themselves for the number of parameters estimated. Using the one-parameter logistic model (1PLM), it is possible to estimate the probability that someone will answer the item (of difficulty, b) correctly. The TwoParameter Logistic Model (2PLM) also aims to estimate the probability of a correct answer, but it also allows estimating the discrimination of the item (a). The bigger the value of "a", the stepper the slope of the ICC and the more discriminant the item is providing. The ThreeParameter Logistic Model (3PLM) estimates the other two parameters described ( $\mathrm{a}$ and $\mathrm{b}$ ) and also the probability for guessing (the " $\mathrm{c}$ " parameter). Imagine that sometimes students get right answers by guessing an alternative in the item. The inclusion of the $\mathrm{c}$ parameter allows the model to estimate the effect of guessing on item difficulty and on examinees final scores, for example. See Hambleton et al. (1991) for a thorough description of these models. This c parameter is especially important with achievement data. However, when our test is composed by other types of items (polytomous, for example), IRT estimation should use other models.

\section{Graded response model}

In this section, we will introduce some equations that represent relevant aspects of the Graded Response Model, as well as, item parameter estimates, and model fit. One of the most popular models used for polytomous items, common with many psychological tests, is the GRM (Samejima F. Estimation of latent ability using a response pattern of graded scores. Psychometrika 1969). This model is appropriate to use when dealing with ordered categories on a rating scale (e.g., a Likert scale reflecting levels of agreement or disagreement) and is considered a generalization of the two-parameter logistic model (2PL) (Keller, 2005). According to Keller, the $2 \mathrm{PL}$ model is used to provide the probability that someone has to receive a specific score (or higher), given the level of the underlying latent trait. The more of the trait (positive affect, for instance) possessed by respondents, the more likely they are to respond with answers that receive higher scores, or the more likely they are to choose one of the more positive ratings on the items of the scale (Hambleton, 2005).

In GRM, the probability $P_{i k}^{*}(\theta)$ that a person's response falls at or above a particular ordered category given $\theta$ (level of latent trait) may be expressed as follows on equation 1 :

$$
P_{i k}^{*}(\theta)=\frac{\exp \left[a_{i}\left(\theta_{j}-b_{i k}\right)\right]}{1+\exp \left[a_{i}\left(\theta_{j}-b_{i k}\right)\right]}
$$

This equation is known as boundary characteristic function of item $i$ for category $k$. The parameter $a_{\mathrm{i}}$ refers to the slope of the function or item discrimination. This parameter is constant for all categories of the same item. Different items will probably present different discriminations though. The $b_{i k}$ parameter, also called threshold parameter, refers to the latent trait level in which the probability of answering at or above the particular category equals $50 \%$ (Hambleton et al. 2011).

GRM considers items as a series of $K-1$ dichotomous items, in which $K$ represents the number of categories in the Likert scale or other ordered category scales. If a 5point scale that goes, say, from 0 to 4 is used, then, the following dichotomies are analyzed for each item: 0 vs. $1,2,3,4 ; 0,1$ vs. $2,3,4 ; 0,1,2$ vs. 3,4 ; and $0,1,2,3$ vs. 4. Because the probability of respondents choosing the lowest category, or any of the higher score categories is $1, P_{i 0}^{*}(\theta)=1$, the threshold parameter for the lowest category is not estimated. Also, the probability of answering above the highest category must be zero, the probability of responding in the highest category is equal to the highest cumulative score category function. The other probabilities based on the other combinations, given $\theta$, are computed by subtracting the adjacent $P_{i k}^{*}$ $(\theta)$. The general expression for this is given on equation 2:

$$
P_{i k}^{*}(\theta)=P_{i k}^{*}(\theta)-P_{i(k+1)}^{*}(\theta)
$$

Or specifically, as presented on equations 3 through 7 :

$$
\begin{aligned}
& P_{i 0}(\theta)=1 \\
& P_{i 1}(\theta)=P_{i 1}^{*}(\theta)-P_{i 2}^{*}(\theta) \\
& P_{i 2}(\theta)=P_{i 2}^{*}(\theta)-P_{i 3}^{*}(\theta)
\end{aligned}
$$




$$
\begin{aligned}
& P_{i 3}(\theta)=P_{i 3}^{*}(\theta)-P_{i 4}^{*}(\theta) \\
& P_{i 4}(\theta)=P_{i 4}^{*}(\theta)
\end{aligned}
$$

Figure 1 presents a graphical representation of the category response functions (CRF) (in terms of probabilities of each rating category over the latent trait scale) for an item and constitutes an example of how we will evaluate negative and positive affect items. The latent trait is conventionally presented in the standardized form that goes from -3 to 3 . The mean of trait scores on the construct of interest is set to 0 and the standard deviation to 1 . These scores can always be transformed later to a more convenient scale (and they would be with most if not all psychological scales). The $a$ parameter for this item is .80 and the $b$ parameters or thresholds for the second, third, fourth and fifth categories are respectively $-2.52,-1.05,-0.05,1.14$. Again, the first category is not estimated since the probability of getting a score of zero or higher is always 1.0. CRF represent the probabilities for responding to each of the five response categories as a function of respondents' level of latent trait (Hambleton, 2005), as, for example, negative and/or positive affect. For example, a person with high levels of negative affect is more likely to choose higher values (as, "this item describes me completely") on the Likert type item that says: "Many times, I feel nervous." CRF can be used to identify items with low category discrimination and spread.

\section{Fitting the graded response model}

To gauge how well the chosen model can predict respondent scores and generate item statistics that are invariant over samples of respondents, it is essential to measure model fit. This involves fitting the GRM to the data, and to estimating item and latent trait parameters.
Usually, different models are tested in order to know which one provides better fit. In this case, another possibility, the partial credit model (Masters, 1982), was not used because it constrains the discrimination power ( $a$ parameter) to be equal for all items. As indicated by one anonymous reviewer, another useful model is the Generalized Partial Credit Model (GPCM: Muraki, 1992) that implements a varying slope parameter and can be used with dichotomous and polytomous items. The GPCM constitutes a useful tool for psychometricians when the categories of Likert-type scales are not spaced. In the educational field, for example, the 0,1 , and 2 points might indicate different degrees of correctness of an answer: 0-incorrect; 1-partial credit; 2-full credit. In the context of affect evaluation, the GPCM might indicate that some categories are more used than others - what does not occur with the GRM that assumes the categories are spaced. A comparison of GPCM and GRM with data from the Affect Scale may result in another useful paper. But it should be noted that often the GPCM and GRM provide very similar results and parameters in the GRM seem easier to explain to practitioners.

The GRM can be fitted using PARSCALE (Muraki \& Bock, 1993). We will provide item parameter estimates and chi-square results as model fit findings (see, Table 1 and Table 2 in the Result section). $\chi^{2}$ statistics are common indicators of model fit, however, some drawbacks need consideration.

Because $\chi^{2}$ statistics are susceptible to sample size, small samples might not reject the null-hypothesis (that states that the item does fit the model) even when the fit is poor. On the other hand, large samples might present significant results even when the fit is appropriate. Therefore, other procedures like evaluating expected and observed scores, as well as, expected and observed residuals (raw and standardized) are considered more

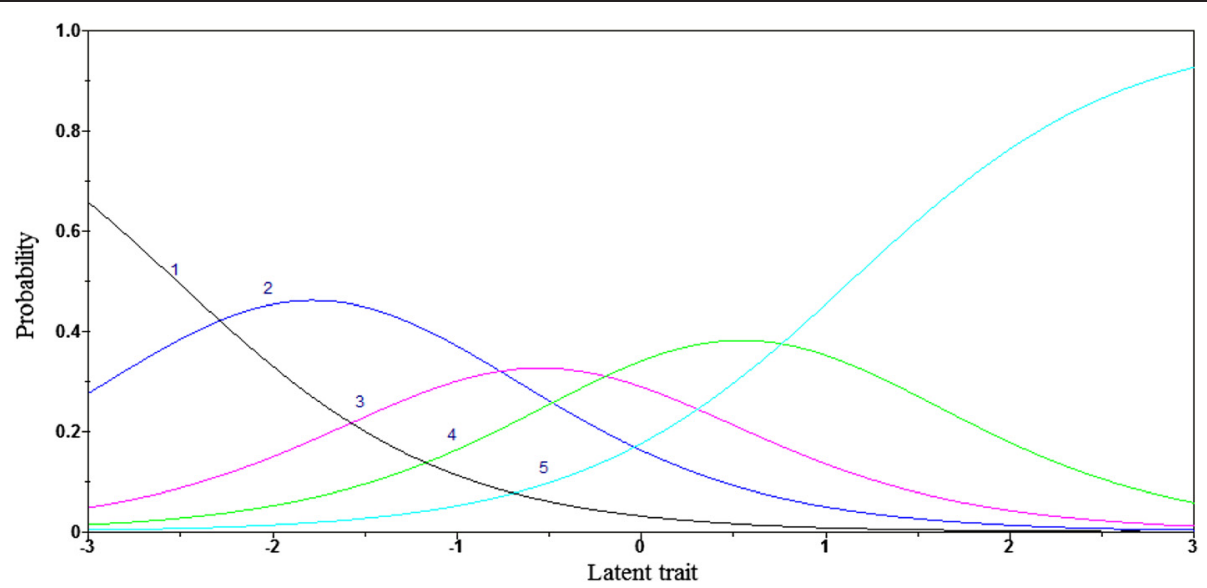

Fig. 1 Category response curves for a five-category graded response model (GRM) item ("Many times, I feel nervous"), with $a=.80, b_{2}=-2.52, b_{3}=-1.05$, $b_{4}=-0.05$, and $b_{5}=1.14$ 
Table 1 Slope, location and chi-square tests for the negative affect scale

\begin{tabular}{lccccc}
\hline Items & Slope $(\mathrm{a})$ & Location $(\mathrm{b})$ & \multicolumn{1}{c}{$x^{2}$} & $\mathrm{df}$ & $p$ \\
\hline 1 & 0.80 & -0.62 & 62.09 & 61 & 0.44 \\
2 & 0.56 & -1.14 & 71.54 & 65 & 0.27 \\
3 & 0.63 & -0.73 & 101.35 & 62 & 0.01 \\
4 & 0.40 & 0.87 & 93.87 & 85 & 0.24 \\
5 & 0.62 & -0.12 & 76.99 & 74 & 0.38 \\
6 & 0.70 & 1.48 & 85.44 & 69 & 0.09 \\
7 & 0.79 & 0.10 & 80.35 & 74 & 0.29 \\
8 & 1.19 & 0.21 & 79.65 & 65 & 0.10 \\
9 & 0.56 & 2.09 & 93.22 & 70 & 0.03 \\
10 & 0.64 & 1.23 & 84.98 & 76 & 0.22 \\
\hline
\end{tabular}

reliable indicators of model fit (Han \& Hambleton, 2007; Liang et al. 2008). Residuals are the difference between expected scores, based on model estimation, and actual results. Standardized residuals are calculated dividing raw residuals by their standard errors. This step was done using ResidPlot-2 (for more details, see Liang et al., 2008).

It is expected that a good fitting model generates standardized residuals (approximately) normally distributed. So, if the model satisfies this assumption, positive evidence of model fit is available. There is no rule of thumb to gauge how "approximately normal" is accepted, so the researcher should take into account the consequences of estimation based on a misfitted model. These consequences might include how many persons will not be selected to be part of a program, or how much a correlation used in research might be underestimated due to poor measurement. At the item level, standardized residuals might be expected between -2 and 2 . Items with residuals out of this interval might indicate poor fit and suggest substitutions in new versions of the test. To evaluate residuals for all categories in each item

Table 2 Slope, location and chi-square tests for the positive affect scale

\begin{tabular}{lcclll}
\hline Items & Slope (a) & Location (b) & $x^{2}$ & $\mathrm{df}$ & $p$ \\
\hline 1 & 1.10 & -0.84 & 65.70 & 49 & 0.06 \\
2 & 0.99 & -1.07 & 71.60 & 45 & 0.08 \\
3 & 0.76 & -2.00 & 45.48 & 42 & 0.33 \\
4 & 0.63 & -2.46 & 33.28 & 36 & 0.60 \\
5 & 0.63 & -1.77 & 59.14 & 45 & 0.08 \\
6 & 1.14 & -1.20 & 42.90 & 39 & 0.31 \\
7 & 0.48 & -2.71 & 68.43 & 43 & 0.01 \\
8 & 0.92 & -1.33 & 68.24 & 42 & 0.01 \\
9 & 1.08 & -0.97 & 40.87 & 44 & 0.61 \\
10 & 0.86 & -1.77 & 52.30 & 38 & 0.06 \\
\hline
\end{tabular}

is also important and might provide worthwhile information about model fit and patterns of answers for each category. For more details about model fit, check the work of Embretson and Reise (2000). The residual evaluations supported the GRM fit with the affect scale.

Another advantage of IRT, when compared with classical methods, is to provide item and test information. Item information functions present the contribution of each item to the latent trait measurement. Items with greater discrimination power contribute more to assessment precision than items with lower discriminating power. This may seem like similar information to point biserial correlations in classical measurement. The big differences are that the IRT statistics are directly linked to measurement precision and the location on the trait scale where that information is relevant. Summing item information functions $\left(I_{i}\right)$ of the test provides a test information function $(I)$, as showed on equation 8 :

$$
I(\theta)=\sum_{i=1}^{n} I_{i}(\theta) .
$$

This characteristic is especially important and unique to item response models because it allows test makers to easily decide which items to choose based on their interests and also based on the impact of the items on the total test information. If the interest is to select respondents that have high levels of the latent trait, items that provide more information on the right hand side of the scale might be chosen to maximize the precision of scores at the higher end of the trait scale.

Test information functions are inversely proportional to standard errors,

$$
S E(\theta)=\frac{1}{\sqrt{I(\theta)}}
$$

So, the more information provided by a test at a particular latent trait, the better the measurement, and the smaller the measurement errors at this level (Hambleton et al., 2000).

\section{The current study}

A common way to conduct research in the field of psychology has been through the use of standardized scales to measure constructs of interest (e.g., affects, optimism, self-esteem). Test development to evaluate positive and negative affect has used the Classical Test Theory (Bradburn, 1969; Fordyce, 1977; Kammann \& Flett 1983; Watson et al. 1988), and, more recently, the Item Response Theory (Ebesutani et al. 2013; Lin \& Yao, 2009). Some reasons why the IRT is still not widely used, despite its advantages, is probably because: a) it requires a deeper statistical background from the researcher (Embretson, 1996), b) it requires large sample size to estimate item and ability rather than CTT - approximately, 
more than 500 examinee responses are requested to achieve proper estimation results, and c) it was more focusing on individual item, and in psychology background, test-level analysis is more reasonable to evaluate with factor analysis (see, Maydeu-Olivares, 2005, for a comparison between IRT and factor analysis). Modern procedures to improve positive and negative affect estimation, however, may contribute to higher discrimination scores among the participants and are critical to the advancement of the field. For this reason, we believe that the readers of the Psicologia Reflexão e Crítica will benefit from a step-to-step description of a sophisticated method to improve test development: the item response theory modeling. Moreover, this paper provides validity and reliability evidences to the Affect Scale (Zanon et al. 2013a) - a standardized test developed to assess positive and negative affect on adults.

What follows is an application of the GRM to the first author's research to develop and evaluate an affect scale. The goal of the next sections is to provide more details about the application, and show how IRT modeling was helpful in the evaluation of positive and negative affect.

\section{Method}

\section{Participants}

The respondents were 853 university students (57\% women and $43 \%$ men) between the ages of 17 and 35 $(\mathrm{M}=21$ years, $\mathrm{SD}=3)$. Participation in the study was voluntary. These numbers are very substantial for IRT research and therefore permit item parameter estimation with a high level of precision.

\section{Instrument}

Affect Scale (Zanon et al. 2013a) is a self report instrument that consists of two independent factors: positive affect and negative affect. Positive and negative affect are characterized by the frequency and intensity of emotions and feelings that an individual experiences as either pleasant or unpleasant (Lyubomirsky et al. 2005). Persons with high scores of positive affect experience frequent and intense episodes of pleasure. They consider themselves happy, enthusiastic, and confident. High levels of positive affect are associated with physical health, conjugal and work satisfaction (Naragon \& Watson, 2009). Conversely, those persons with high levels of negative affect experience repeated episodes of intense displeasure (Watson, 2005). Generally, these respondents perceive themselves as sad, discouraged, and anxious. In general, high levels of negative affect are associated with rumination (Trapnell \& Campbell, 1999), anxiety, depression (Miles et al. 2004), and neuroticism (Zanon et al. 2013b).

Each factor (positive and negative) consists of 10 sentences representing the various moods and emotions of persons, such as "I am proud of myself", and "I have been worried lately". Each statement is rated by the respondents on a 5-point Likert scale. The rating scale is defined by the end points: 1 - this item does not describe me at all, and 5 - this item describes me completely. The higher the number, the more the item describes the respondent, and the lower the number, the less the item describes the respondent.

\section{Procedures}

Respondents responded collectively to the questionnaires from within their classrooms. Beforehand, the students were informed that participation in the study was voluntary, and that all information shared would remain confidential. The students were asked to carefully read the questions and respond according to their personal feelings. Data collection was performed in a single session for each class of students. This study is part of a project approved by Ethics Committee and all ethical procedures were followed on this research.

\section{Results}

\section{Internal structure evidence}

Factor analysis (with an oblimin rotation) identified two principal factors that explained $38.4 \%$ of the total variance $(25.6 \%$ for positive affect and $12.8 \%$ for negative affect). All items had positive factor loadings between .46 and .74 in the first dimension, and between .38 and .77 on the second one. These items presented loadings lower than .40 in the other dimension. Coefficient alpha was .83 for positive affect and .77 for negative affect and the correlation between the factors was -.38. These results were gathered with the same sample used for the IRT analysis and indicate preliminary evidences of validity and reliability for the Affect Scale.

\section{IRT modeling}

IRT analyses were conducted for the positive and negative scale separately. From the correlational information, it seemed clear that the two scales were rather different. Table 1 presents slopes (a), location (b) and chi-square tests for the negative affect scale. The slopes were considered moderate. The location parameters indicate that the items were spread over the continuum of the scale. Five items were on the left hand side of the scale (below the mean respondent score) and the other five were on the right hand side (above the mean). Chi-square tests showed that two items were significant, which suggest that these items were not well fitted by the model. However, residual inspection (at the test, item and category level) provided enough evidence to consider that the model actually fitted the item level data. Table 2 presents slopes (a), location (b) and chi-square tests for the positive affect scale. The slopes were of moderate size, and 
slightly higher than the other scale, which meant that the positive affect items were generating more item level and test level information. All items were located on the left hand side of the scale suggesting that many respondents were finding it easy to identify with the statements. Two items presented significant chi-squares, but again, residual analyses suggested that the model fit was acceptable.

\section{Residual analyses}

Raw and standardized scores were analyzed at the item level. Initially, the amount of answers for each category (e.g., 1, 2, 3, 4 and 5) in every single item is compared with the amount of expected answers -produced by the chosen model. Categories without answers or with large discrepancies between observed and expected values indicate poor fit. Another procedure is to evaluate how small the residuals (differences between the observed and expected scores from each item) are on the latent trait continuum for each items. An item, for example, might present small residuals on the low part of the continuum, but large residuals on the rest - this also would indicate poor fit. Visual inspection of the residuals on all categories and for each item was conducted for the negative and positive affect.

Residual analyses were interpreted graphically. ResidPlot2 provides confidence bounds for the expected raw residuals on the latent trait continuum for an arbitrary number of intervals (13 in this study), and also provides standardized residuals (plotted on 13 intervals) spread over the latent trait. For raw residuals, good fitting model is verified when observed residuals are found between the confidence bounds; while for standardized residuals, good fitting model is seen when the plots are between -2 and 2 .

At the category level, raw and standardized residuals for the five categories of each item were analyzed. For the raw residuals, this step focused on the observed residuals out of the confidence bounds, and for the standardized residuals, the number of plots out of -2 and 2 were considered. Regarding the negative affect scale, approximately, $58 \%$ of all categories presented observed residuals between the bounds in all 13 intervals; and $56 \%$ of all categories presented standardized residuals between -2 and 2 . For the remaining categories, no more than two observed and standardized residuals were found out of the confidence bounds and -2 and 2 respectively in each category. At the item level, similar procedures were conducted. And, most of the observed and standardized residuals again were on the confidence bounds and between -2 and 2 . At the test level, observed and expected standardized residual distribution for all items were assessed, along with observed and expected score distribution. Visual inspection revealed small differences, which suggested acceptable fit.

Regarding the positive affect scale, approximately, $48 \%$ of all categories presented observed residuals between the bounds in all intervals; and $38 \%$ of all categories presented standardized residuals between -2 and 2. No more than three observed and standardized residuals were found out of the confidence bounds and -2 and 2 respectively in each category. At the item level, most of the observed and standardized residuals were on the confidence bounds and between -2 and 2; and for all items, again small differences were found between observed and expected standardized residuals and score distribution, conferring acceptable fit to the scale.

\section{Test information functions}

Figures 2 and 3 present test information functions (solid lines) and standard errors (broken lines) to the positive and negative scales, respectively. As shown in Fig. 2, most of the information provided by the scale was below the mean of respondent scores suggesting that the scale was better designed for respondents with lower scores.

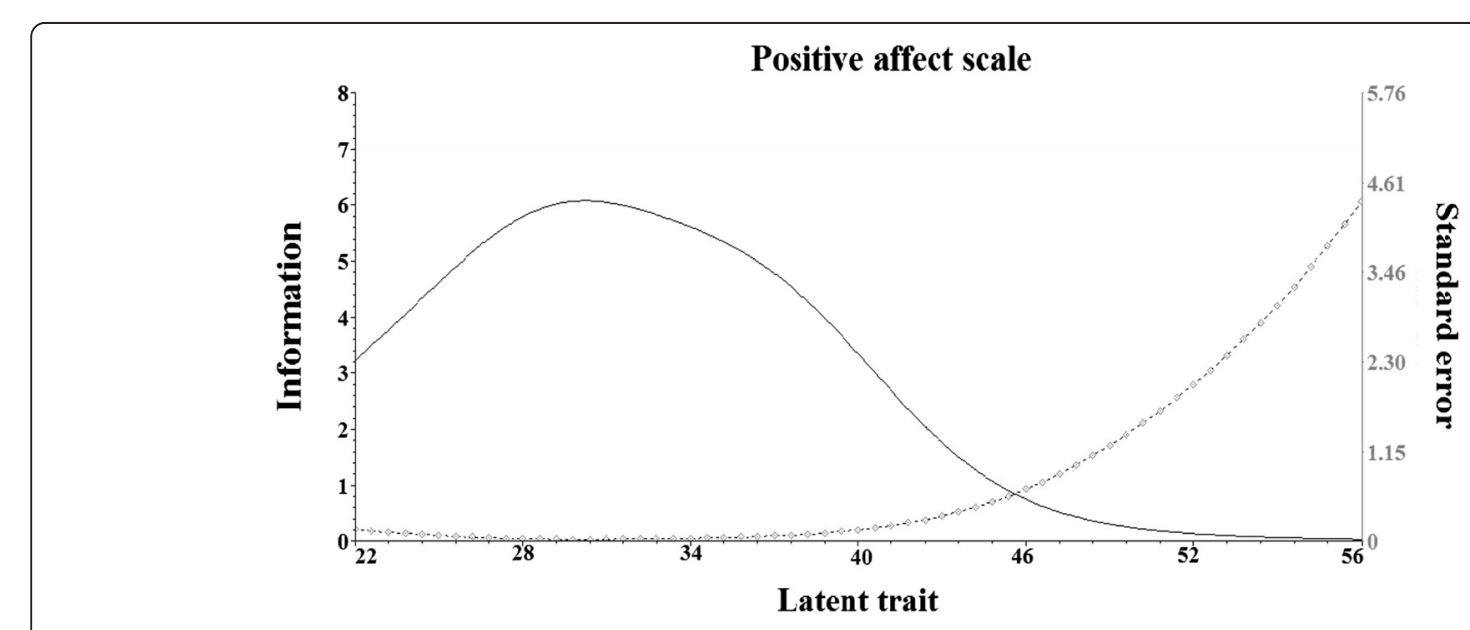

Fig. 2 Test information function (solid line) and standard errors (broken line) to the positive affect scale 


\section{Negative affect scale}

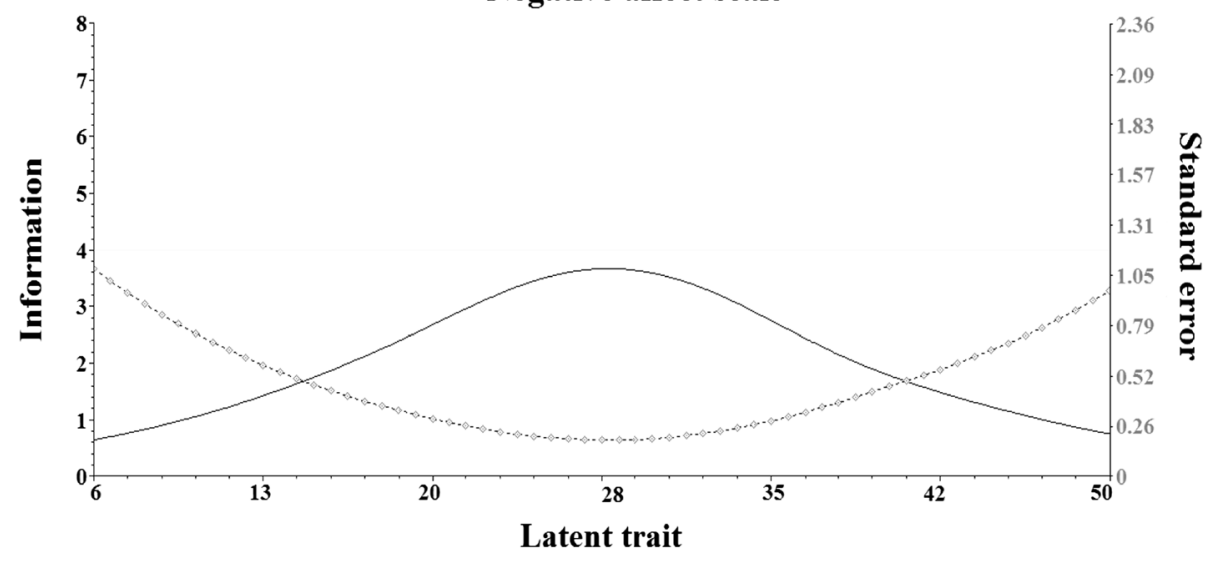

Fig. 3 Test information function (solid line) and standard errors (broken line) to the negative affect scale

Regarding the negative affect scale, the information was well spread over the continuum. However, the amount of information given was much lower than the positive affect scale and less than what was considered acceptable. In a redesign, perhaps the clarity of the negative statements could be improved, and perhaps the addition of a few more items could increase the level of test information. But historically, negatively worded items have always proved more problematic for respondents.

\section{Discussion}

The two most important advantages provided by an IRT application during the development and analyses of these scales are probably item and ability parameter invariance and test information functions. Many psychological tests used to assess personality, intelligence, attitudes etc. might also benefit from these IRT advantages. In research as well as in applied contexts, details about the area in which the latent traits measured by the test are doing better measurement (and how well) would help clinics, researchers or test makers to decide which test is more appropriate for their specific interests. In addition, a test that is free of examinee sample characteristics in test development allows for more reliable comparisons among respondents as well as groups (see some recent IRT applications in the Brazilian context: Brant et al. 2015; Carvalho et al. 2013; Knijnik et al. 2014; Lopes, \& Bueno, 2014; Muniz et al. 2014).

Often, classical methods exclusively are used to offer evidence of validity and reliability to new tests and this evidence is undoubtedly important. But this paper provides evidence that IRT results can be extremely helpful to complement this evidence with information regarding the quality of the measurement at specific points of the measuring scale. CTT can provide this same information but it is more cumbersome to do so using little known statistics for assessing conditional errors. In this case, it is clear that the positive affect scale should not be used for the purpose of evaluating people with high levels of positive affect. The amount of information provided by the 10 items is simply not very good at the higher score levels. For this situation to change, more "difficult" items are needed in the scale-items that would be difficult for respondents to agree with and therefore permit better assessment at the high end of the score continuum. Situations in which people are being selected based on their (high level of) intelligence or other skills/traits might be misleading, unfair, and even unreliable, if the information function and errors provided by the test presented similar distribution and values to those shown in Fig. 2.

IRT applications provide different standard errors of measurement at different trait levels. Because standard errors of measurement are used in score interpretations, it is possible to easily create confidence intervals to interpret individual scores (Embretson, 1996). So, one can have a range (around the reached score) associated with a probability. The smaller the errors at some level, the smaller the confidence bands. This does not happen with classical methods that nearly always assume the same standard error applies at all trait levels.

As shown in Fig. 3, the quality of the measurement provided by the negative affect scale is spread over the continuum and it is relatively higher at the middle of the scale (around the mean). However, the amount of information provided is relatively low and reflects items with modest discriminative indices. It raises the question of how well these statements were actually written. Perhaps they were confusing to respondents. Due to parameter invariance properties, these items will keep their psychometric characteristics in other applications. So, new items can be included in future revisions of these scales and a reevaluation of the item information functions could be conducted. These procedures can be repeated until one gets the desired test. 
According to Embretson (1996), psychologists have had little exposure to IRT especially because it is both relatively new and statistically sophisticated (compared with classical methods), measurement courses have declined in graduate programs of psychology so not only are graduate students less knowledgeable about CTT, they may have little or no knowledge of IRT, and because not much understandable material has been focused on psychological issues (most of them are to educational assessment). The latter point was a focal point within this paper. Two introductory books are suggested to students and professors interested in IRT (for psychological examples, see Embretson \& Reise, 2000; for educational examples, see Hambleton et al., 1991).

This study is not free of limitations. Differential item functioning (DIF) constitutes a serious threat to test validity and needs to be further addressed on the items of the Affect Scale. DIF occurs when participants with identical latent trait provide different patterns of answers to some items. This might be the case of items that favor (or disfavor) some sample's subgroups (e.g., girls and boys, black and white, minorities). Considering that women are more likely than men to present depression symptoms, the investigation of DIF across sex on items of positive and negative affect might reveal possible differences on these groups.

\section{Conclusion}

Lastly, IRT is not a panacea and does not correct problems of misleading items or failure to meet content specifications. It also does not substitute classical methods that were influential and are still important. However, IRT is a worthwhile tool that can and should be used to increase the quality of psychological tests and consequently psychological assessment.

\section{Endnotes}

${ }^{1}$ As indicated by one anonymous reviewer, this might not be the case for Rasch models because is expected that the data fit the model and not the opposite. Our position, however, is that models that do not fit the data are of little value and removing items from the test to fit the model can decrease its content validity. The Rasch model also assumes that test items are all equally discriminating and this assumption runs counter to the widely held view that test items in practice often differ in both their difficulty levels and discriminating powers.

${ }^{2}$ Measurement invariance depends on the degree of representativeness of the sample in the population being studied. Thus, parameter invariance might not hold for samples with extremely different characteristics.

${ }^{3}$ There are IRT models that are multidimensional and do not require evidence of unidimensionality. See Reckase (2009) for multidimensional IRT models, and Kose and Demirtasli (2012) for a comparison between uni and multidimensional IRT models.

\section{Competing interests}

The authors declare that they have no competing interests.

\section{Authors' contributions}

CZ participated on the study conception, writting, statistical analysis, data interpretation, and revision. CSH participated on writting, data interpretation, and revision. $\mathrm{HY}$ participated on statistical analysis, data interpretation and revision. RKH participated on the study conception, writting, statistical analysis, datainterpretation, and revision. All authors read and approved the final manuscript.

\section{Author details}

${ }^{1}$ Department of Psychology, Universidade São Francisco, Alexandre Rodrigues Barbosa Str. 45, Itatiba 13251-900SP, Brazil. ²Department of Psychology, Federal University of Rio Grande do Sul, Ramiro Barcelos Str. 2600, Porto Alegre 90035-003, RS, Brazil. ${ }^{3}$ School of Education, University of Massachusets Amherst, Hills South, USA.

Received: 26 November 2015 Accepted: 7 April 2016

Published online: 18 April 2016

\section{References}

Bradburn NM. The structure of psychological well being. Chicago: Aldine Publishing; 1969.

Brant SRC, Pilati R, Borges-Andrade JE. Estratégias de aplicação do aprendido: análise baseada em TRI. Psicologia: Reflexão e Crítica. 2015;28(1):01-10. doi:10.1590/1678-7153.201528101.

Carvalho LF, Monteiro RM, Alcará AR, Santos AAA. Aplicação da TRI em uma medida de avaliação da compreensão de leitura. Psicologia: Reflexão e Crítica. 2013;26(1):47-57. doi:10.1590/S0102-79722013000100006.

Ebesutani C, Regan J, Smith A, Reise S, Chorpita BF, Higa-McMillan C. The 10-item positive and negative affect schedule for children, child and parent shortened versions: Application of item response theory for more efficient assessment. J Psychopathol Behav Assess. 2013;34:191-203. doi:10.1007/s10862-011-9273-2.

Embretson SE. The new rules of measurement. Psychol Assess. 1996;8(4):341-9. doi:10.1037/1040-3590.8.4.341.

Embretson SE, Reise SP. Item Response Theory for Psychologists. Mahwah: Lawrence Erlbaum Associates; 2000.

Fordyce MW. Development of a program to increase personal happiness. J Couns Psychol. 1977;24:511-21. doi:10.1037//0022-0167.24.6.511.

Gulliksen H. Theories of Mental Test Scores. New York: Wiley; 1950.

Hambleton RK. Item response theory: A broad psychometric framework for measurement advances. Psicothema. 1994;6(3):535-56.

Hambleton RK. Applications of item response theory to improve health outcomes assessment: Developing item banks, linking instruments, and computer-adaptive testing. In: Lipscomb J, Gotay CC, Snyder C, editors. Outcomes Assessment in Cancer: Measures, Methods and Applications. Cambridge: Cambridge University Press; 2005. p. 445-64.

Hambleton RK, Jodoin M. Item response theory: models and features. In: Fernández-Ballesteros R, editor. Encyclopedia of Psychological Assessment. London: Sage; 2003. p. 509-14.

Hambleton RK, Robin F, Xing D. Item response models for the analysis of educational and psychological test data. In: Tinsley HEA, Brown SD, editors. Handbook of Applied Multivariate Statistics and Mathematical Modeling. San Diego: Academic; 2000. p. 553-85.

Hambleton RK, Swaminathan H, Rogers HJ. Fundamentals of Item Response Theory. Thousand Oaks: Sage Publications; 1991.

Hambleton RK, van der Linden WJ, Wells CS. IRT models for the analysis of polytomous scored data: Brief and selected history of model building advances. In: Nering ML, Ostini R, editors. Handbook of Polytomous Item Response Theory Models. New York: Routledge; 2011. p. 21-42.

Han KT, Hambleton RK. User's Manual: WinGen (Center for Educational Assessment Report no. 642). Amherst: University of Massachusetts, Center for Educational Assessment; 2007

Hattie J. Methodology review: Assessing unidimensionality of tests and items. Appl Psychol Meas. 1985;9:139-64.

Horn J. A rationale and test for the number of factors in factor analysis. Psychometrika. 1965;30(2):179-85. 
Kammann R, Flett R. A scale to measure current level of general happiness. Aust Psychol. 1983:35(1):259-65.

Keller LA. Item response theory (IRT) models for polytomous response data. In: Everitt BS, Howell DC, editors. Encyclopedia of Statistics in Behavioral Sciences. Chichester: John Wiley \& Sons; 2005. p. 990-1003.

Knijnik LF, Giacomoni CH, Zanon C, Stein LM. Avaliação dos subtestes de leitura e escrita do teste de desempenho escolar através da Teoria de Resposta ao Item. Psicologia: Reflexão e Crítica. 2014;27(3):481-90. doi:10.1590/1678-7153. 201427308 .

Kose A, Demirtasli NC. Comparison of one-dimensional and multi-dimensional models based on item response theory in terms of Both variables of test sample and length size. Proceeding - Social and Behavioral Sciences. 2012;46: 135-40. doi:10.1016/j.sbspro.2012.05.082.

Liang T, Han KT, Hambleton RK. User's Guide for ResidPlots-2: Computer Software for IRT Graphical Residual Analyses, Version 2.0 (Center for Educational Assessment Research Report no. 688). Amherst: University of Massachusetts, Center for Educational Assessment; 2008.

Lin TH, Yao G. Evaluating Item Discrimination Power of WHOQOL-BREF from an Item Response Model Perspectives. Soc Indic Res. 2009;91:141-53. doi:10.1007/s11205-008-9273-0.

Lopes J, Bueno M. Construção e validação de uma prova de Matemática para alunos do $1^{\circ}$ ao $4^{\circ}$ ano de escolaridade. Psicologia: Reflexão e Crítica. 2014; 27(3):434-42. doi:10.1590/1678-7153.201427303.

Lord, F. M. A theory of test scores. Psychometrika 1952; Monograph 7.

Lord FM, Novick MR. Statistical Theories of Mental Test Scores. Reading: AddisonWesley; 1968.

Lorenzo-Seva U, Timmerman ME, Kiers HAL. The Hull method for selecting the number of common factors. Multivar Behav Res. 2011;46(2):340-64. doi:10.1080/00273171.2011.564527.

Lyubomirsky S, King L, Diener E. The benefits of frequent positive affect: Does happiness lead to success? Psychol Bull. 2005;131(6):803-55. doi:10.1037/0033-2909.131.6.803.

Maydeu-Olivares A. Linear IRT, non-linear IRT, and factor analysis: A unified framework. In: Maydeu-Olivares A, McArdle JJ, editors. Contemporary Psychometrics. A Festschrift to Roderick P. McDonald. Mahwah: Lawrence Erlbaum; 2005. p. 73-100.

Masters GN. A Rasch model for partial credit scoring. Psychometrika. 1982;47(2): 149-74. doi:10.1007/bf02296272.

Miles $\mathrm{H}$, MacLeod AK, Pote $\mathrm{H}$. Retrospective and prospective cognitions in adolescents: Anxiety, depression, and positive and negative affect. J Adolesc. 2004;27(6):691-701. doi:10.1016/j.adolescence.2004.04.001.

Muniz M, Rueda FJM, Nery JCS. Analysis of psychometric properties of the cube test to evaluate visuospatial reasoning. Psicologia: Reflexão e Crítica. 2014 27(3):504-14. doi:10.1590/1678-7153.201427311.

Muraki E. A generalized partial credit model: Application of an EM algorithm. Appl Psychol Meas. 1992;16(2):159-76. doi:10.1177/014662169201600206.

Muraki E, Bock RD. PARSCALE: IRT-Based Test Scoring and Item Analysis for Graded and Open-Ended Exercises and Performance Tasks. Chicago: Scientific Software International; 1993.

Naragon K, Watson D. Positive affectivity. In: Lopez SJ, editor. Encyclopedia of Positive Psychology, vol. 2. Malden: Wiley-Blackwell; 2009. p. 707-11.

Reckase MD. Multidimensional Item Response Theory: Statistics for Social and Behavioral Sciences. New York: Springer; 2009.

Rupp AA, Zumbo BD. A note on how to quantify and report whether IRT parameter invariance holds: when Pearson correlations are not enough. Educ Psychol Meas. 2004;64(4):588-99. doi:10.1177/0013164403261051.

Samejima F. Estimation of latent ability using a response pattern of graded scores. Psychometrika 1969; Monograph 7.

Trapnell PD, Campbell JD. Private self-consciousness and the five-factor model of personality: distinguishing rumination from reflection. J Pers Soc Psychol. 1999:76(2):284-304. doi:10.1037//0022-3514.76.2.284

Watson D. Positive affectivity: The disposition to experience pleasurable emotional states. In: Snyder CR, Lopez SJ, editors. Handbook of Positive Psychology. New York: Oxford University Press; 2005. p. 106-19.

Watson D, Clark LA, Tellegen A. Development and validation of brief measures of Positive and Negative Affect: The PANAS Scales. J Pers Soc Psychol. 1988; 54(1):1063-70. doi:10.1037/0022-3514.54.6.1063.

Yu CH, Popp SO, DiGangi S, Jannasch-Pennell A. Assessing unidimensionality: A comparison of Rasch modeling, Parallel Analysis, and TETRAD. Practical Assessment, Evaluation \& Research. 2007;12(14):1-19.
Zanon C, Bastianello MR, Pacico JC, Hutz SC. Development and validation of a positive and negative affect scale. Psico-USF. 2013a;18(2):193-202. doi:10.1590/s1413-82712013000200003.

Zanon C, Bastianello MR, Pacico JC, Hutz SC. Relationships between positive and negative affect and the five factors of personality in a Brazilian sample. Paidéia (Ribeirão Preto). 2013b;23(56):285-92. doi:10.1590/1982-43272356201302.

\section{Submit your manuscript to a SpringerOpen ${ }^{\circ}$ journal and benefit from:}

- Convenient online submission

- Rigorous peer review

- Immediate publication on acceptance

- Open access: articles freely available online

- High visibility within the field

- Retaining the copyright to your article

Submit your next manuscript at springeropen.com 